\title{
PRENATAL DIAGNOSIS OF A NEW CASE: DE NOVO BALANCED NON-ROBERTSONIAN TRANSLOCATION INVOLVING $\mathrm{t}(15 ; 22)(\mathrm{p} 11.2 ; \mathrm{q} 11.2)$
}

\author{
Atli EI $\dot{I}^{1, *}$, Gurkan $\mathrm{H}^{1}$, Atli E ${ }^{1}$, Tozkir $\mathrm{H}^{1}$, Varol $\mathrm{GF}^{2}$, İnan $\mathrm{C}^{2}$ \\ *Corresponding Author: Emine İkbal Atli, Ph.D., Department of Medical Genetics Faculty of Medicine \\ Trakya University, Edirne, Turkey. Tel: +90-554-253-40-30. Fax: +90-284-223-33-14. \\ Email: emine.ikbal@gmail.com
}

\begin{abstract}
The balanced non-Robertsonian translocation (ROB) associated with acrocentric chromosomes is an unusual phenomenon. We report the case of rare non-ROB involving chromosomes 15 and 22 with cytogenetic and molecular cytogenetic findings of 46,XY,t(15;22)(p11.2;q11.2). To the best of our knowledge, $t(15 ; 22)$ is the first report of this breakpoint that is not the usual non-ROB. The karyotype of the chorionic villus cell was 46,XY,t(15;22)(p11.2; q11.2) from two different initial cultures. This is different from the usual non-ROB of acrocentric chromosomes. Comparative genomic hybridization has been performed to determine the chromosomal origin. Non-Robertsonian translocation associated with acrocentric chromosomes is an unusual event and only a few cases have been reported. In this study, we observed acrocentric chromosomes 15 and 22 as a rarely balanced non-ROB, where satellites of chromosome 15 translocated to chromosome 22 and part of chromosome 22 were translocated to chromosome 15 . To the best of our knowledge, our patient is the first case reported in the literature for this translocation in prenatal and postnatal periods.
\end{abstract}

Keywords: Array comparative genomic hybridization (array-CGH); Chorion villus sampling (CVS); Cytogenetics; Fluorescence in situ hybridization (FISH); Non-Robertsonian translocation (non-ROB).

\footnotetext{
${ }^{1}$ Department of Medical Genetics, Faculty of Medicine, Trakya University, Edirne, Turkey

${ }^{2}$ Department of Gynecology and Obstetrics, Faculty of Medicine, Trakya University, Edirne, Turkey
}

\section{INTRODUCTION}

Robertsonian translocations (ROBs) are the most common balanced chromosomal abnormalities in the population involving (often nonhomologous and rarely homologous) acrocentric chromosomes with an prevalence of 1.23/1000 live births. Balanced translocations were determined at $0.2 \%$ in the neonatal population, $0.6 \%$ in infertile couples and $9.2 \%$ in cases of recurrent miscarriages [1]. Phenotypically, carriers of balanced reciprocal translocation will be normal. These individuals have a high reproductive risk of having abnormal embryos due to chromosomal imbalances during meiosis, leading to the birth of affected offspring or recurrent miscarriages. Balanced non-ROB involving acrocentric chromosomes is a rare event. We document the case of rare non-ROB involving chromosomes 15 and 22 with cytogenetic and molecular cytogenetic finding 46,XY,t(15.22)(p11). To the best of our knowledge, $\mathrm{t}(15 ; 22)$ is the first documented anomaly with this uncommon breakpoint. With the aim to determine the chromosomal starting place of the $46, \mathrm{XY}, \mathrm{t}(15 ; 22)$ (p11.2; q11.2) translocation, array comparative genomic hybridization $(\mathrm{CGH})$ was performed. To the best of our knowledge, this is the first documented report of a fetus with molecular characterization of a $\mathrm{t}(15 ; 22)(\mathrm{p} 11.2 ; \mathrm{q} 11.2)$, present as a de novo non-Robertsonian balanced translocation.

Case Presentation. The proband, a 26-year-old woman was referred for chorion villus sampling (CVS) at 12 weeks' gestation because of a history of pregnancies with abnormal ultrasound findings (nuchal fold thickness). Nuchal fold thickness of the fetus was measured as $4.25 \mathrm{~mm}$. Hyperechogenic focus was detected in the right 
ventricle of the fetus. A fetal right choroid plexus cyst was measured at $4.5 \mathrm{~cm}$. The proband had a dental X-ray during the 5 th week of her pregnancy. The proband was informed and then signed the consent form for the invasive prenatal sampling and cultivation of the chorionic villus biopsy specimen. A transabdominal CVS was performed. Chromosome analysis was done as per standard technique. Leishmann-pancreatin banding metaphases with 500 band resolution were analyzed for chromosome identification. A minimum of 20 metaphases was evaluated in the proband and parents. The chorionic villus cells karyotype was $46, X Y, t(15 ; 22)(\mathrm{p} 11.2 ; \mathrm{q} 11.2)$ from two different initial cultures.

This translocation is different from the usual ROB of acrocentric chromosomes. The satellite region of chromosome 15 translocated to long arm of chromosome 22 and part of chromosome 22 translocated to chromosome 15 (Figure 1).

The rearranged chromosomes were further characterized by array-CGH, fluorescent in situ hybridization (FISH) and a silver-staining technique for nucleolar organizer regions (Ag-NOR). According to the karyo-

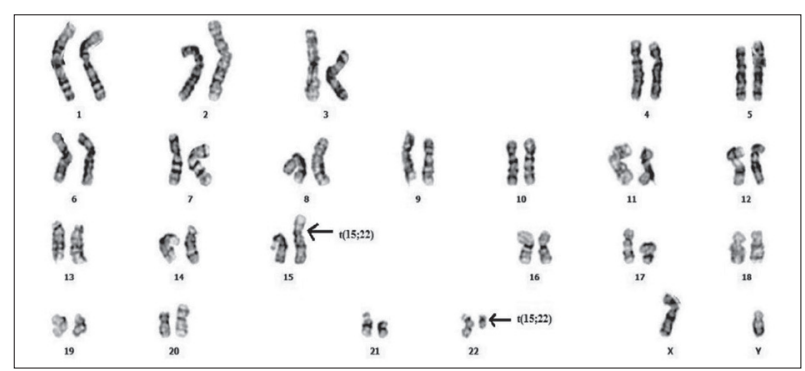

Figure 1. The proband's karyotype: $\mathrm{t}(15 ; 22)(\mathrm{p} 11.2 ; \mathrm{q} .11 .2)$.

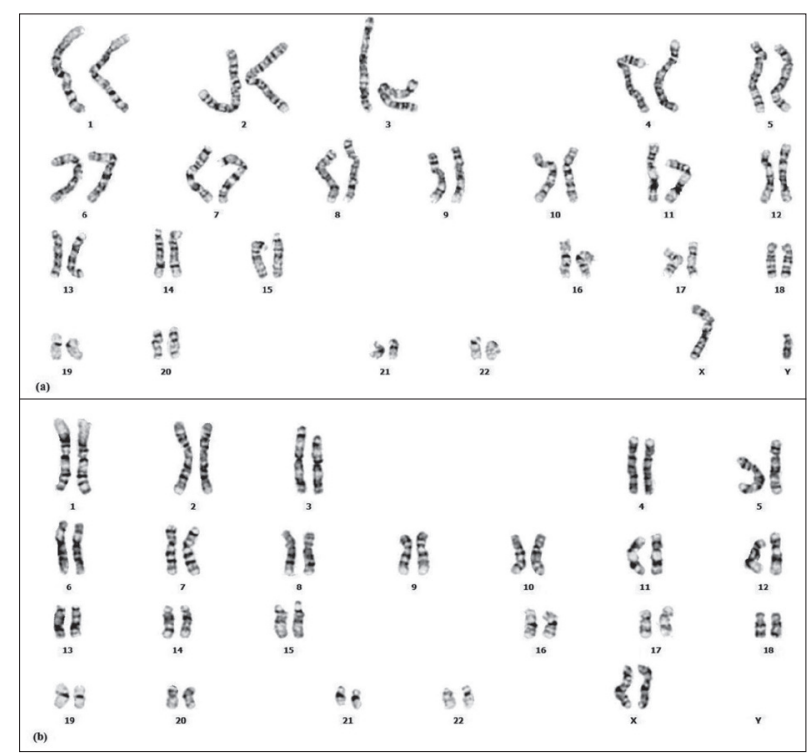

Figure 2. The parents' karyotype: (a) 46,XY; (b) 46,XX. type and array results, the proband was a balanced carrier. Array-comparative genomic hybridization (Agilent 180k; Agilent Technologies, Santa Clara, CA, USA) was performed according to the manufacturer's instructions. A resolution of at least $100 \mathrm{~kb}$ was achieved with array (Figure 2). The Ag-NOR banding was performed by adding two drops of $50.0 \%$ silver nitrate and $2.0 \%$ gelatin on slides, respectively. The slides were then sealed with cover glasses and incubated at $60{ }^{\circ} \mathrm{C}$ for $5 \mathrm{~min}$. Subsequently, the slides were soaked in distilled water until the cover glasses were separated and were then stained with 20.0\% Giemsa solution (Sigma-Aldrich Chemie $\mathrm{GmbH}$, Taufkirchen, Germany) for $1 \mathrm{~min}$. Fluorescent in situ hybridization was performed as required by the routine protocol. A rapid FISH analysis was performed on interphase cells (uncultured chorionic villus sample). We exclusively utilized an FDA-approved FISH test kit for rapid aneuploidy screening in uncultured chorionic villus sample. The Aquarius ${ }^{\circledR}$ Prenatal Enumeration Kit (Cytocell Technologies, Cambridge, Cambridgeshire, UK) consisted of three satellite DNA probes for chromosome enumeration probes X, Y, and 18 (CEP X, CEPY, and CEP 18) and two locus-specific identifier probes for 13q14 (LSI13) and 21q22.13-22.2 (LSI 21). The three centromeric probes and the two locus-specific probes were applied to the samples in two separate hybridizations on the glass slide (Cytocell Technologies). Results were enumerated on the counting of 50 interphase nuclei per target and are reported as the number. The parents were healthy and consanguineous (children of cousins) and had no family history of genetic disorders or congenital malformations. They had a healthy 10-month-old boy from the first pregnancy. Karyotype on peripheral lymphocytes was performed on both parents and the results were normal (Figure 3).

Written informed consent was obtained from the patient to have the case details published. Ethics approval and consent to take part was received from the mother and father of the baby to have the case details and accompanying images published.

\section{DISCUSSION AND CONCLUSIONS}

De novo structural chromosome rearrangements will be considered in three major categories: apparently balanced rearrangements, including reciprocal translocations, ROB and inversions. Apparently unbalanced rearrangements. Small supernumerary chromosomes. The finding of an apparently balanced de novo chromosome rearrangement in amniocytes poses a counseling dilemma.

A significant excess of all balanced de novo rearrangements (about eight times the newborn incidence) was observed in individuals with intellectual deficiency. For 


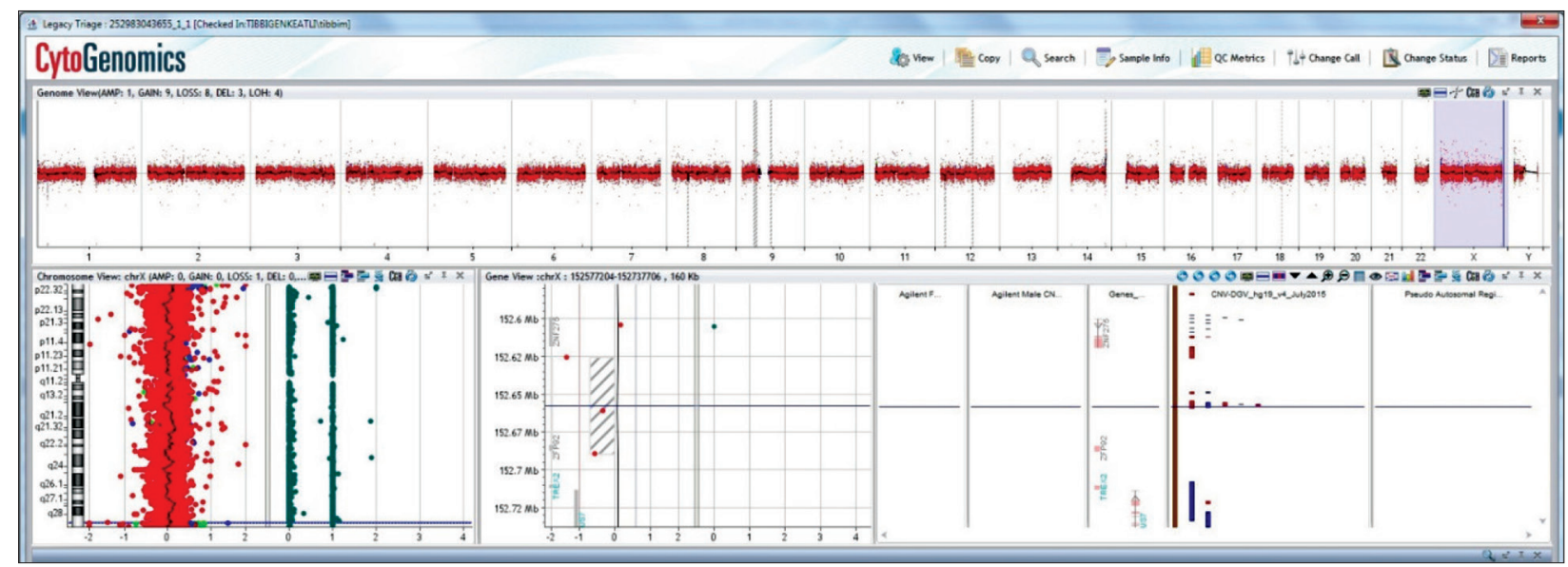

Figure 3. Array comparative genomic hybridization of the chorionic villus sample image is normal (180k array; Agilent Technologies).

each 10,000 invasive prenatal genetic process, six to nine cases with a de novo balanced rearrangement and four to nine cases with a de novo unbalanced rearrangement may be expected [2].

Non-Robertsonian translocation involving acrocentric chromosomes is a rare event, and only a few cases have been reported. In the present study, we observed acrocentric chromosomes 15 and 22 as a rare balanced non-ROB, where satellites of chromosome 15 translocated to chromosome 22 and part of chromosome 22 was translocated to chromosome 15. Mangelschots et al. [3] reported a case with $46, \mathrm{XX}, \mathrm{t}(13 ; 15)(\mathrm{q} 12 ; \mathrm{q} 13)$ where she gave birth to a child with karyotype $46, X X,+13,+\operatorname{der}(15) \mathrm{t}(13 ; 15)(\mathrm{q} 12$; q13)mat. Most of the ROBs are balanced in relation to acrocentric chromosomes with the breakpoints (q10; q10).

Rupa et al. [4] described a couple, 35-year-old wife and 37-year-old husband, with the history of two miscarriages and one congenitally abnormal fetus. The couple was physically and intellectually normal. Chromosome analysis revealed normal male karyotype in the husband, whereas the female showed a balanced reciprocal trans-location as 46,XX,t(13;15)(p11.2;q22.1). Frikha et al. [5] reported a familial non-ROB between the long arm of chromosome 15 and the long arm of chromosome 21 with $46, \mathrm{XY}, \mathrm{t}(15 ; 21)$ (q21;q21) in an infertile man and his hypo-fertile brother whose wife had a history of repeated pregnancy loss.

Only the nuchal fold thickness increase was reported in the prenatal period of our case. Balanced reciprocal translocations are generally harmless rearrangements for the carriers, however, the derivative chromosomes and their matching homologous form a quadrivalent parent at meiosis I and 2:2, 3:1, and 4:0 segregation models yield in most cases of unbalanced gametes [6,7]. To the best of our knowledge, our proband is the first and unique case reported in the literature for this translocation in both prenatal and postnatal periods. In conclusion, in the couples with abnormal fetal sonography findings, cytogenetic analyses and chromosome-specific FISH paintings should be performed in the prenatal period. While providing genetic counseling, pre implantation genetic diagnosis could be suggested.

\section{ACKNOWLEDGMENTS}

We kindly thank the all clinicians for their participation in this study. Please contact the author for any further information.

Declaration of Interest. The authors report no conflicts of interest. The authors alone are responsible for the content and writing of this article.

\section{REFERENCES}

1. Moorhead PS, Nowell PC, Mellman WJ, Battips DM, Hungerford DA. Chromosome preparations of leukocytes cultured from human peripheral blood. Exp Cell Res. 1960; 20(3): 613-616.

2. Peter PA. Prenatal diagnosis of chromosomal abnormalities through chorionic villus sampling and amniocentesis. In: Milunsky A, Milunsky JM, Editors. Benn Genetic Disorders and the Fetus Diagnosis, Prevention, and Treatment, 7th ed. Hoboken, NJ, USA: Wiley Blackwell; 2015: 178-266.

3. Mangelschots K, Van Roy B, Speleman F, Van Roy $\mathrm{N}$, Gheuens J, Beuten J, et al. Reciprocal translocation between the proximal regions of the long arms of chromosomes 13 and 15 resulting in unbalanced offspring: Characterization by fluorescence in situ hybridization and DNA analysis. Hum Genet. 1992; 89(4): 407-413.

4. Rupa D, Neeraja K, Deepak C, Swarna M. A rare balanced nonrobertsonian translocation involving acrocentric chromosomes: Chromosome abnormality of 
t(13;15)(p11.2; q22.1). J Hum Reprod Sci. 2016; 9(2): 128-130.

5. Frikha R, Turki F, Daoud S, Kaabi O, Louati R, Trabelsi $\mathrm{O}$, et al. A rare familial non Robertsonian translocation involving chromosomes 15 and 21 and failure of reproduction: Is there a correlation? (2809S). Presented at the 64th Annual Meeting of the American Society of Human Genetics, San Diego, CA, USA, October 19 2014. Am Soc Hum Genet. 2014; 95(3): 214-221.
6. Kayhan G, Ergün MA, Biri AA, Karaoğuz MY. A prenatal tertiary trisomy resulting from balanced maternal 8;9 translocation. J Turk Ger Gynecol Assoc. 2011; 12(3): 183-185.

7. Sheth F, Liehr T, Shah K, Sheth J. Prader-Willi syndrome - type 1 deletion, a consequence of an unbalanced translocation of chromosomes 13 and 15, easily to be mixed up with a Robertsonian translocation. Mol Cytogenet. 2015; 8: 52. 